\title{
Liver failure-future challenges and remaining questions
}

\author{
Peter Lemmer, Josef Christian Pospiech, Ali Canbay^ \\ Department of Medicine, Ruhr University Bochum, University Hospital Knappschaftskrankenhaus Bochum, Bochum, Germany \\ Contributions: (I) Conception and design: A Canbay; (II) Administrative support: None; (III) Provision of study material or patients: None; (IV) \\ Collection and assembly of data: P Lemmer, JC Pospiech; (V) Data analysis and interpretation: All authors; (VI) Manuscript writing: All authors; (VII) \\ Final approval of manuscript: All authors. \\ Correspondence to: Ali Canbay, MD. Professor of Medicine, Director of the Department of Internal Medicine, Ruhr University Bochum, In der \\ Schornau 23-25, 44892 Bochum, Germany. Email: ali.canbay@rub.de.
}

\begin{abstract}
This review compiles the current state of controversial aspects of liver failure and outlines future challenges. The definition of acute liver failure (ALF) is widely accepted and implies no previous liver injury whereas the term "acute-on-chronic liver failure" remains contested. We will promote a concept, in which we differentiate three types of liver failure: ALF, acute-on-chronic liver failure (AOCLF) and acuteon-liver-cirrhosis (AOCi). The mechanistical insights into the coagulation system in patients with hepatic insufficiency have increased fundamentally in the past 10 years. Therefore, we follow now the concept of the so-called rebalanced hemostasis. This lower-level equilibrium arises from the fact that most coagulation factors and inhibitors are synthesized within the liver. We will demonstrate the advantage of viscoelastic test methods, which can assess the coagulation situation in patients with liver insufficiency much more precisely than conventional global coagulation tests. The therapeutic option of emergency liver transplantation (ELT) has significantly improved the prognosis of patients with ALF. However, limiting factors such as shortage of organs increase more and more the need for reliable prognostic markers. Due to a better understanding of the regenerative process during ALF new survival markers and prognostic tools have been emerging on the horizon in the last decade. Therefore, we will describe the current state of research in this field.
\end{abstract}

Keywords: Hepatic insufficiency; liver failure; prediction of prognosis; rebalanced hemostasis; survival markers

Submitted Jun 26, 2020. Accepted for publication Jan 18, 2021.

doi: $10.21037 /$ atm-20-4968

View this article at: http://dx.doi.org/10.21037/atm-20-4968

\section{Introduction}

An acute injury to the liver, which cannot be compensated by the regenerative capacity results in acute and rapid hepatocyte death and subsequently impairs liver function. This clinical syndrome is termed acute liver failure (ALF) and is associated with high mortality in the absence of immediate state-of-the-art intensive care and/or emergency liver transplantation (ELT). ALF is defined as coagulopathy [International Normalized Ratio (INR) >1.5] and any grade of hepatic encephalopathy (HE) in a patient without pre-existing liver disease (1). The Acute Liver Failure Study Group in the USA categorizes ALF as hyperacute
( $<7$ days), acute (7-28 days) and subacute (28 days to 6 months) depending on latency between onset of symptoms and development of coagulopathy and encephalopathy. In general, higher latencies are associated with higher mortality.

While precise figures on the incidence of ALF in Germany are still lacking, the collective experience of various transplant centers leads us to assume 200 to 500 cases annually in this country. It should be noted that there has been a shift in the predominant causes of ALF. In the early 1980s acute hepatitis $B$ virus (HBV) infection was viewed as the most frequent cause, but in the last four decades ALF due to toxic

^ ORCID: 0000-0001-6069-7899. 
liver injury has increased dramatically. This includes direct/ intrinsic and idiosyncratic reactions. ALF caused by viral hepatitis (HAV, HEV), autoimmune disease (or hepatitis), ischemia, pregnancy, Wilsons disease, and congestive heart failure still occur. The cause remains unidentified in roughly $15 \%$ of adults and $50 \%$ of children with ALF (2).

ALF is characterized by the massive death of hepatocytes due to different types of cellular demise (e.g., necrosis, apoptosis, etc.). This loss of cells ultimately leads to functional impairment of the liver. Not only the predominant mechanism of cell death but also the prognosis varies depending on the underlying etiology. In recent years a lot of progress has been made in the comprehension of the unique capacity of the liver to recover. As an attempt to restore hepatic architecture and function several intrinsic regenerative processes are simultaneously triggered. When ALF is not fatal, a complete recovery of the liver and its function is possible. However, the question remains under which circumstances the regenerative processes can sufficiently cope with sudden, confluent hepatocyte death and subsequent loss of function. If regeneration cannot compensate for cell loss, the only curative option remaining is ELT (3).

The liver plays a crucial role in blood coagulation as most coagulation factors are synthesized and cleared by liver parenchymal and reticuloendothelial cells, respectively (4). Mainly for this reason patients with liver diseases, both acute and chronic, exhibit complex coagulation disorders involving changes in pro- and anticoagulation factors, as well as changes in fibrinolysis, leading to both bleeding and thrombosis (5). Although a lot of progress has been made in this field, several aspects still remain unclear. For example, it is unknown whether the coagulation status might be changing in the course of ALF e.g., by a switch in the conformation of coagulation proteins.

The intention of this review is to describe controversial aspects in liver failure and thus outline future challenges and remaining questions. In this context we will discuss the definition of ALF, aspects of coagulation, and prediction of prognosis. Current literature was retrieved by a selective search of PubMed/Medline listed manuscripts.

\section{How should liver failure be defined depending on pre-existing liver injury?}

Although there are still differences in the detailed clinical diagnosis of ALF, there is a broad consensus among the societies EASL, AASLD and APASL that four clinical features are mandatory: elevated transaminases, an elevated bilirubin level, an impaired coagulation (INR $>1.5$ ) and the presence of HE. Furthermore, it is widly accepted that the definition of ALF implies no previous liver injury. Acutely decompensated cirrhosis and acute-on-chronic liver failure (AOCLF) are two regularly encountered clinical conditions observed in patients with known chronic liver disease. The term "acutely decompensated cirrhosis" is widely accepted and refers to the development of ascites, encephalopathy, gastrointestinal hemorrhage, or any combination of these disorders in patients with cirrhosis. In recent years there have been many controversies concerning the term "Acuteon-chronic liver failure", a term originally suggested by Jalan and Williams (6).

This definition is misleading. Indeed, AOCLF is not identical to acute-on-cirrhosis liver failure (AOCi). Chronic liver disease (injury) is associated with elevated liver enzymes in most cases but not obviously impaired liver function. Patients with chronic liver disease don't exhibit spider naevi, esophageal varices or splenomegaly, which are considered clear signs of liver cirrhosis. In contrast, in compensated liver cirrhosis, serum liver enzymes can be well within normal ranges, but the patients may already show signs of portal hypertension. The most important difference between AOCLF and AOCi is the liver regeneration capacity and thus the probability of survival. Hence, we advocate for a more clear definition for these variants of liver failure (Figure 1).

In contrast to the original definition, we would like to promote an alternative concept in which the definition of AOCLF comprises patients with previous liver disease but excludes the ones with liver cirrhosis. In one of our recent studies, we compared ALF patients to AOCLF patients (without cirrhosis) to identify possible clinically relevant separators (7). We found that ALF and AOCLF had similar clinical outcomes. However, we identified differences in the causes of liver failure and in possible risk factors for outcome. The most common causes for liver failure were pharmacological-toxic effects in ALF patients and viral infections in AOCLF patients.

In summary, we suggest a definition of AOCLF as liver failure, with pre-existing liver injury or disease (independent of the cause) without cirrhosis and no prior decompensation events. Patients with cirrhosis suffering from an additional acute injury, independent of decompensation events, would be grouped as AOCi. ALF would include only patients with no previous liver injury.

Since the patients with these three different types of 


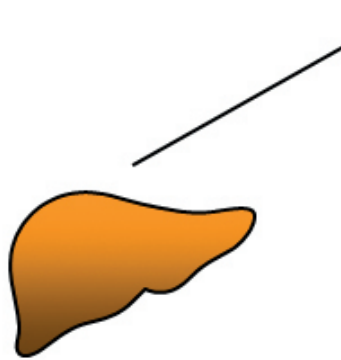

Acute

(ALF)

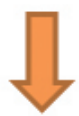

NO previous liver disease

e.g., acute viral hepatitis, toxic liver injury

High regenerative capacity

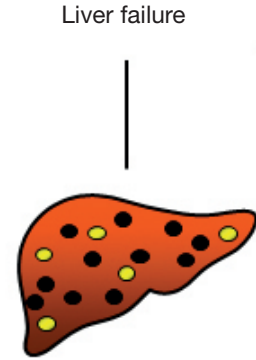

Acute-on-chronic (AOCLF)

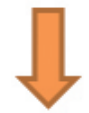

Pre-existing liver injury without cirrhosis

e.g., NAFLD, ALD, HBV, HCV

Impaired regnerative capacity

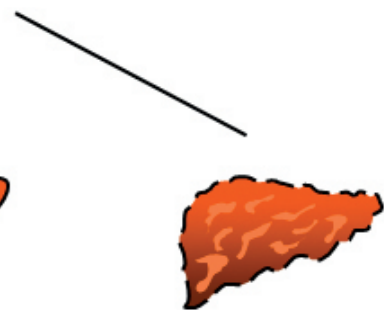

Acute-on-cirrhosis (AOCi)

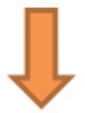

Pre-existing cirrhosis

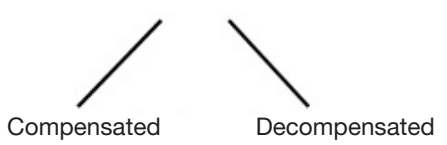

No regenerative capacity

Figure 1 Schematic representation of different types of liver failure according to pre-existing liver injury. The here presented alternative definition categorizes liver failure upon an acute injury based on the type of pre-existing liver injury by chronic liver disease. When no pre-existing liver injury is present an ALF is given. When an acute injury occurs in addition to pre-existing chronic liver disease, without cirrhotic alterations, an acute-on-chronic liver failure (AOCLF) is given, which exhibits only slightly worse outcome but different requirement for patient handling than ALF. If an acute injury occurs in an already cirrhotic liver this leads to acute-on-cirrhosis liver failure (AOCi), which has worse outcome and requires immediate intensive care support due to lack of any regenerative capacity of the liver. ALF, acute liver failure.

liver failure differ in many aspects, e.g., with respect to the precipitating events or the risk stratification, this patient heterogeneity should have an impact on the design of clinical intervention trials. In this context, post-hoc exploratory data analysis could be helpful in generating meaningful hypotheses for further studies.

\section{Are the classic coagulation parameters associated with bleeding complications in liver failure?}

In the past 10 years understanding of the coagulation system in patients with hepatic insufficiency has improved fundamentally. In these cases, we now follow the concept of the so-called rebalanced hemostasis. Since coagulation factors and inhibitors alike are synthesized within the liver the result is lower-level equilibrium of coagulation.
The quantitative reduction of coagulation factors in liver patients correlates with the severity of the disease and is shown in classic coagulation parameters and global tests (INR, aPTT, fibrinogen, platelets). For example, increased INR values-due to historical reasons-suggest a tendency to bleed. They are often incorrectly used to assess the risk of bleeding before endoscopic interventions, in a surgical setting, but also in patients suffering from hepatic insufficiency. However, this does not take into account the fact that anticoagulant factors are reduced at the same time.

Hence, the classic global tests do not correlate with the actual bleeding risk in patients with hepatic insufficiency. Thus, the INR should only be considered as a synthesis parameter (8). In the majority of patients with liver failure a shift in hemostasis to a procoagulatory condition can be assumed. Unnecessary transfusion of coagulation factors in these situations is not only costly, it may also induce 
complications such as portal vein thrombosis and other thrombotic events. Viscoelastic test methods (VET) such as the ROTEM $^{\circledR}$ analysis represent a rather novel development to improve the clinical management of patients with different types of liver failure. VET methods have already been applied in the perioperative care of patients with different coagulation disorders. These methods allow a more precise assessment of the coagulation status, which can be used to determine whether fibrinogen, platelets or prothrombin complex are required, or whether fibrinolytic therapy is required. VET methods have the additional advantage, that they can be used as "Point-of-care" (POC) method and are therefore faster than conventional laboratory diagnostics (9).

In patients with AOCLF, it has already been demonstrated that the amount of substituted coagulation factors before minimally invasive interventions can be significantly reduced by using the ROTEM ${ }^{\circledR}$ analysis compared to conventional laboratory tests. No increased rate of bleeding episodes or thrombotic complications was observed under this approach (10). However, it should be noted that the largest data base for patients suffering from hepatic insufficiency is currently available in the context of liver transplantation (LT) (reducing the need for transfusion). Hence, clinical application of this promising method in the various forms of liver failure, as defined above, needs to be based on further prospective studies.

In summary, the advantage of VET is based on its ability to assess the coagulation situation in patients with liver insufficiency much more precisely than the conventional global tests. Although this method is very promising and increasingly used, it is still too early for a final recommendation, since further studies are needed to confirm its benefit regarding their use on the various forms of liver failure. Another important area of coagulation research should focus on the role of different cell types of the liver. An interesting question would be to clarify the role of the hepatic stellate cells (HSC) or Kupffer cells in production or activation of pro- and anticoagulation factors.

\section{Short- and long-term prognosis of ALF outcome depend on different clinical factors}

As we have pointed out before ALF can take a fulminant course and is associated with high mortality. The therapeutic option of ELT has significantly improved the prognosis of these patients. However, applicability of ELT is limited by organ shortage, utilization of large amounts of healthcare resources, and graft rejection in a considerable proportion of cases. More reliable prognostic parameters to identify patients who would either recover without ELT or who would not benefit from ELT at all could improve resource utilization and outcome for patients. Currently for evaluation of prognosis in ALF King's College (KCC) and Clichy criteria are broadly established, universally recognized tools. Both systems have been expanded for different ALF etiologies in the past. However, accuracy of both systems in predicting the patients' outcome is limited. In particular identifying patients who will survive without LT still remains a major challenge (11). In the following paragraphs we give an overview on a few alternative markers which could expand or complement available prognostic tools.

Another prognostic tool is the Model for End-Stage Liver Disease (MELD), which exhibited better prognostic accuracy than KCC and Clichy criteria (12). While not all pathophysiologic mechanisms in ALF are understood, apoptosis, necrosis, and necroapoptosis are a hallmark of ALF (13). In a previous study we could show that replacing bilirubin with M65, a marker for overall cell death, could improve prediction of fatal outcomes when calculating the MELD (14). An overview of currently used scoring systems is given in Table 1.

ALF triggers a regenerative response of remaining healthy hepatocytes. Subsequently resident liver progenitor cells (LPC) become activated to support, or take over the role of regeneration. Depending on the individual regenerative capacity some patients will recover spontaneously from ALF, while in many cases, this capacity cannot compensate the amount of hepatocyte loss. In these cases, LT offers the only potential hope for survival. Cumulative data hint to activation of the LPC compartment, not only in chronic liver disease, but generally, when confluent loss of hepatocyte mass occurs. Indeed, LPC activation correlates with severity of liver injury, and clinical outcomes in ALF. The degree of LPC response could be a potential biomarker of regenerative capability in ALF.

ELT and LT in general have significantly improved short term survival in ALF to over $80 \%$ after 1 year and $>70 \%$ after 5 years (15). This improved outcome after LT is due to multimodal therapy concepts, standardized evaluation of organ recipients and donors, improved organ storage, optimized surgical methods and perioperative management, and development of specific immunosuppressive agents with reduced side effects. However, long-term prognosis after LT is still highly variable and depends on a large 
Table 1 Scoring systems for severity of acute liver failure/necessity of transplantation and novel candidate factors

\begin{tabular}{|c|c|c|}
\hline \multicolumn{2}{|c|}{ Scoring system/criteria/candidate } & $\begin{array}{l}\text { Evaluation/prognostic factors } \\
\text { Arterial } \mathrm{pH}<7.25 \text { (independent of stage of hepatic encephalopathy) } \\
\text { OR two out of three of the following criteria and clinical deterioration: } \\
\text { - INR }<6.5 \\
\text { - creatinine }>300 \mu \mathrm{mol} / \mathrm{L} \\
\text { - hepatic encephalopathy grade } 3-4\end{array}$ \\
\hline & Other causes & $\begin{array}{l}\text { INR }>6.5 \text { (independent of hepatic encephalopathy) OR } \\
\text { three out of five of the following criteria (independent of stage of encephalopathy): } \\
\text { - age }<10 \text { or }>40 \text { years } \\
\text { - etiology: unclear, medication-toxic } \\
\text { - time from icterus to encephalopathy }>7 \text { days } \\
\text { - INR }>3.5 \\
\text { - Bilirubin }>300 \mu \mathrm{mol} / \mathrm{L}\end{array}$ \\
\hline MELD & & $10 \times\left(0.957 \times \ln n_{\text {serum creatinine }}+0.378 \times \ln n_{\text {bilirubin }}+1.12 \times I n_{I N R}+0.643\right)$ \\
\hline CK-18/modified MELD & & $10 \times\left(0.957 \times \ln n_{\text {serum creatinine }}+0.378 \times \ln n_{\text {serum CK-18/M65 }}+1.12 \times \ln n_{I N R}+0.643\right)$ \\
\hline BILE score & & $\begin{array}{l}\text { Bilirubin }(\mu \mathrm{mol} / \mathrm{L}) / 100+\text { lactate }(\mathrm{mmol} / \mathrm{L}) \\
+4 \text { (for cryptogenic ALF, Budd-Chiari syndrome, or } \\
\text { phenprocoumon toxicity) } \\
-2 \text { (for acetaminophen toxicity) } \\
\pm 0 \text { (for other etiologies of ALV) }\end{array}$ \\
\hline ALFSG Index & & Coma grade, Bilirubin, INR, phosphorus, $\ln n_{M з о}$ \\
\hline ALFED Model & & $\begin{array}{l}\text { Dynamic of variables over } 3 \text { days: } \\
\text { HE 0-2 points; INR 0-1 point; } \\
\text { Arterial ammonia 0-2 points; serum Bilirubin 0-1 point }\end{array}$ \\
\hline
\end{tabular}

variety of factors. metabolic and cardiovascular diseases, impaired renal function, infections, recurrence of the underlying disease/cause, and development of malign tumors. In addition, graft rejection remains a relevant reaction after LT limiting the prognosis, although a lot of progress has been made in recent years to optimize and individualize immunosuppressive therapy. Such reactions always require histological evaluation and should be classified following the Banff Working Group on Liver Allograft Pathology (16). A major challenge after LT is to adjust immunosuppression to control and avoid graft rejection. Though, immunosuppressive substances worsen factors, which determine long-term prognosis as risk for recurrence of underlying disease, development of de novo malignancies, and cardiovascular risk profile (increased risk for type 2 diabetes, hyperlipidemia, obesity) (17). Most immunosuppressants are also nephrotoxic. Thus, to further improve long-term survival after LT for ALF it will be necessary to improve adaptation of immunosuppressant therapy and to identify the individually ideal therapy regimen.

\section{Is fibrosis necessary for liver regeneration?}

It is widely accepted that continuous hepatocyte death due to chronic liver injury (e.g., due to viral hepatitides, chronic ethanol intoxication, drug abuse, or obesity) promotes liver fibrosis over the course of chronic liver diseases (18). The 
chronic damage to hepatocytes results in activation of HSCs into contractile myofibroblasts. Activated HSC produce collagen to stabilize liver architecture and to assist tissue repair (19). In the long term this can lead to cirrhosis and further sequelae (liver cancer; hepatic failure). However, fibrosis could be interpreted as beneficial wound healing process in acute injury settings, which is supported by data indicating that fibrosis may be reversed upon the removal of the damaging agent (20). We could demonstrate that in ALF profibrotic processes occur in parallel to increased HSC activity. We also observed increased liver stiffness in ALF patients by transient elastography (21), which was correlated with the degree of liver cell damage and HSC activation. This supports our hypothesis that fibrosis occurs not only in chronic liver disease, but also in ALF presumably as a proregenerative mechanism. Studies in larger cohorts of patients will be required to analyze if transient elastography or fibrosis markers might serve as new prognostic tools for ALF.

\section{What are novel developments in diagnosis and therapy of liver failure?}

Among the most pressing issues in the clinical handling of ALF are diagnostic options, that would give more information on remaining liver function and regenerative capacity. One method that is relatively novel is the LiMax, which can estimate liver function from a breath test. In particular, enzymatic activity of specific cytochrome c enzymes is measured by detection of their products in the breath of patients (22). In ALF this method seems to allow more accurate measurement of liver function than conventional serum tests and may enable monitoring of ALF course or even prediction of survival without LT (23).

Therapeutic options in ALF are limited to LT or are dependent on the specific causes of ALF (2). Experimental therapies as plasma exchange or blood purification (also termed liver dialysis) did not have significant benefits for transplant free survival or recovery time in studies performed up to date (24). The evidence for plasma exchange is rather limited and a clinical benefit is in question. Liver dialysis can reduce serum bilirubin and bile acid concentrations (25), however, it is unclear if some bile acid species might be required for liver regeneration. Thus, liver dialysis may on the one hand remove harmful bile acids while on the other hand also reducing signals for regeneration. One way to support liver regeneration could be to supply the liver with stem cells to renew regenerative capacity. Preliminary data from adipocyte derived stem cell transplantation in alcoholinduced ALF look promising and could be expanded for other etiologies (26).

\section{Challenges of LT for ALF}

Standard procedure for treatment of patients with ALF is intensive care with close monitoring and early continuous veno-venous haemodiafiltration in case of kidney failure. Antibiotic or antimycotic therapy should only be applied, when an infection is present. Supplementation of coagulation factors must be critically evaluated as it is usually unnecessary. This procedure is aimed to support the patient until the regenerative capacity of the affected liver can restore function. The only curative option, when recovery from ALF seems unlikely is (orthotopic) LT. However, LT comes with several limitations on its own. The first and most obvious problem is organ shortage in most societies, where many more patients wait for a suitable transplant liver than organs are available. For ALF there are certain criteria that allow high urgency listing, which can somewhat compensate for the organ shortage in very critical cases, though this again depends on the actual availability of donor organs. Thus, it is imperative to prevent a progression of ALF into a highly critical situation and also to develop better algorithms for detection of patients, who would not require LT to recover. All scores currently in use to assess the urgency of LT in ALF (King's college criteria, Clichy criteria, MELD) lack in the identification of patients, who have sufficient regenerative capacity to recover without LT. This is a major challenge of ALF in the near future to improve on these scores or develop a new algorithm to exclude those patients from LT, who have a good prognosis. Apart from these options further improvement and research has to go into specific treatment of ALF by etiology. There are certain causes of ALF (i.e., paracetamol intoxication, autoimmune related, HBV-associated) where direct, specific treatment according to the causing agent can avoid LT and improve outcome. It would be highly desirable to develop such options for other etiologies. Finally, patients who received LT will require life-long immunosuppression, which poses a risk for infections and other complications. Current clinical research is aiming to develop personalized and well-adjusted immunosuppression therapy, which would increase long-term survival and well-being of LT recipients. Taken together the major challenges in LT for ALF are identification of patients acutally not requiring LT and improving non-LT therapy for ALF to avoid this 
drastic option.

\section{Summary and conclusions}

An acute injury to the liver, resulting in acute and rapid hepatocyte death and subsequently impaired liver function is termed ALF. Although a lot of progress in the comprehension of this syndrome has been made in recent years, there are still some controversial aspects remaining. This review was aimed to describe the current state of science in these research areas and to outline future challenges.

The definition of ALF is widely accepted and implies no previous liver injury whereas the term "acute-on-chronic liver failure" (AOCLF) remains contested. In contrast to patients with ALF, patients with AOCLF cannot currently be included in the ELT list. This limitation is based on a definition, in which AOCLF patients already suffer from liver cirrhosis. The patients with AOCLF in our definition have a pre-existing liver damage with no cirrhosis and have a better prognosis.

In the context of coagulation disorders involving changes in coagulation factors and inhibitors, VET methods represent promising approaches regarding an improvement of the clinical management of patients with different types of liver failure. Although this method is increasingly used, further studies are needed to confirm its benefit in the therapeutic decision process.

The therapeutic option of ELT has significantly improved the prognosis of ALF patients. Limiting factors such as shortage of organs and overstrained healthcare resources continuously increase the need for reliable prognostic parameters. An improved understanding of the regenerative process during liver failure and cell death mechanisms could yield novel markers for survival and more prospective and multi-centric studies are required to assess performance of novel markers.

\section{Acknowledgments}

Funding: This project was supported by the Deutsche Forschungsgemeinschaft (DFG CA267/13-3) and the Wilhelm Laupitz Foundation to AC.

\section{Footnote}

Provenance and Peer Review: This article was commissioned by the Guest Editors (Ralf Weiskirchen and Wolfgang
Stremmel) for the series "Unresolved Basic Issues in Hepatology" published in Annals of Translational Medicine. The article has undergone external peer review.

Peer Review File: Available at http://dx.doi.org/10.21037/ atm-20-4968

Conflicts of Interest: All authors have completed the ICMJE uniform disclosure form (available at http://dx.doi. org/10.21037/atm-20-4968). The series "Unresolved Basic Issues in Hepatology" was commissioned by the editorial office without any funding or sponsorship. AC serves as an unpaid editorial board member of Annals of Translational Medicine from Aug 2020 to Jul 2022. The authors have no other conflicts of interest to declare.

Ethical Statement: The authors are accountable for all aspects of the work in ensuring that questions related to the accuracy or integrity of any part of the work are appropriately investigated and resolved.

Open Access Statement: This is an Open Access article distributed in accordance with the Creative Commons Attribution-NonCommercial-NoDerivs 4.0 International License (CC BY-NC-ND 4.0), which permits the noncommercial replication and distribution of the article with the strict proviso that no changes or edits are made and the original work is properly cited (including links to both the formal publication through the relevant DOI and the license). See: https://creativecommons.org/licenses/by-nc-nd/4.0/.

\section{References}

1. Lee WM, Squires RH Jr, Nyberg SL, et al. Acute liver failure: Summary of a workshop. Hepatology 2008;47:1401-15.

2. Canbay A, Tacke F, Hadem J, et al. Acute liver failure: a lifethreatening disease. Dtsch Arztebl Int 2011;108:714-20.

3. Best J, Dollé L, Manka P, et al. Role of liver progenitors in acute liver injury. Front Physiol 2013;4:258.

4. Bedreli S, Sowa JP, Malek S, et al. Rotational thromboelastometry can detect factor XIII deficiency and bleeding diathesis in patients with cirrhosis. Liver Int 2017;37:562-8.

5. Tripodi A, Mannucci PM. The coagulopathy of chronic liver disease. N Engl J Med 2011;365:147-56.

6. Arroyo V, Moreau R, Jalan R. Acute-on-Chronic Liver Failure. N Engl J Med 2020;382:2137-45. 
7. Gottlieb A, Kottmann M, Manka P, et al. How to Define Acute Liver Failure Patients with Pre-Existing Liver Disease without Signs of Cirrhosis. Dig Dis 2019;37:147-54.

8. Tripodi A, Primignani M, Mannucci PM, et al. Changing Concepts of Cirrhotic Coagulopathy. Am J Gastroenterol 2017;112:274-81.

9. Weber CF, Zacharowski K, Brün K, et al. Basic algorithm for Point-of-Care based hemotherapy: perioperative treatment of coagulopathic patients. Anaesthesist 2013;62:464-72.

10. Bedreli S, Sowa JP, Gerken G, et al. Management of acuteon-chronic liver failure: rotational thromboelastometry may reduce substitution of coagulation factors in liver cirrhosis. Gut 2016;65:357-8.

11. Choi WC, Arnaout WC, Villamil FG, et al. Comparison of the applicability of two prognostic scoring systems in patients with fulminant hepatic failure. Korean J Intern Med 2007;22:93-100.

12. Yantorno SE, Kremers WK, Ruf AE, et al. MELD is superior to King's college and Clichy's criteria to assess prognosis in fulminant hepatic failure. Liver Transpl 2007;13:822-8.

13. Bechmann LP, Marquitan G, Jochum C, et al. Apoptosis versus necrosis rate as a predictor in acute liver failure following acetaminophen intoxication compared with acute-on-chronic liver failure. Liver Int 2008;28:713-6.

14. Bechmann LP, Jochum C, Kocabayoglu P, et al. Cytokeratin 18-based modification of the MELD score improves prediction of spontaneous survival after acute liver injury. J Hepatol 2010;53:639-47.

15. Olivo R, Guarrera JV, Pyrsopoulos NT. Liver Transplantation for Acute Liver Failure. Clin Liver Dis 2018;22:409-17.

Cite this article as: Lemmer P, Pospiech JC, Canbay A. Liver failure-future challenges and remaining questions. Ann Transl Med 2021;9(8):734. doi: 10.21037/atm-20-4968
16. Ormonde DG, de Boer WB, Kierath A, et al. Banff schema for grading liver allograft rejection: utility in clinical practice. Liver Transpl Surg 1999;5:261-8.

17. Anastácio LR, Ribeiro Hde S, Ferreira LG, et al. Incidence and risk factors for diabetes, hypertension and obesity after liver transplantation. Nutr Hosp 2013;28:643-8.

18. Canbay A, Friedman S, Gores GJ. Apoptosis: the nexus of liver injury and fibrosis. Hepatology 2004;39:273-8.

19. Parola M, Marra F, Pinzani M. Myofibroblast - like cells and liver fibrogenesis: Emerging concepts in a rapidly moving scenario. Mol Aspects Med 2008;29:58-66.

20. Friedman SL, Bansal MB. Reversal of hepatic fibrosis -fact or fantasy? Hepatology 2006;43:S82-8.

21. Dechêne A, Sowa JP, Gieseler RK, et al. Acute liver failure is associated with elevated liver stiffness and hepatic stellate cell activation. Hepatology 2010;52:1008-16.

22. Stockmann M, Lock JF, Malinowski M, et al. The LiMAx test: a new liver function test for predicting postoperative outcome in liver surgery. HPB (Oxford) 2010;12:139-46.

23. Buechter M, Gerken G, Hoyer DP, et al. Liver maximum capacity (LiMAx) test as a helpful prognostic tool in acute liver failure with sepsis: a case report. BMC Anesthesiol 2018;18:71.

24. Larsen FS, Schmidt LE, Bernsmeier C, et al. High-volume plasma exchange in patients with acute liver failure: An open randomised controlled trial. J Hepatol 2016;64:69-78.

25. Donati G, Angeletti A, Gasperoni L, et al. Detoxification of bilirubin and bile acids with intermittent coupled plasmafiltration and adsorption in liver failure (HERCOLE study). J Nephrol 2021;34:77-88.

26. Götze T, Krueger M, Meutsch J, et al. Three Cases of Alcohol-Induced Acute-On-Chronic Liver Failure With Successful Support by Adipose-Derived Stem Cells. Clin Transl Gastroenterol 2019;10:e00095. 